\title{
A two-scale model for discrete cell gravure roll coating
}

\author{
R.W.Hewson ${ }^{1}$, N.Kapur and P.H.Gaskell \\ Institute of Engineering Thermofluids, Interfaces and Surfaces \\ School of Mechanical Engineering \\ The University of Leeds \\ Leeds LS2 9JT
}

\begin{abstract}
A two-dimensional model for predicting the fluid pick-out and coated film thickness characteristics of a discrete-cell direct gravure roll coater operating in reverse mode is derived. A novel multi-scale approach is adopted for this purpose and the resulting equations solved numerically for inertia-less flow conditions. A system of stiff ordinary differential equations is found to be sufficient to capture the major gross flow features, while at the cell level the analysis is based on a finite element solution of the momentum and continuity equations. It represents the first such predictive model of its kind, with particular interest placed on the nature of both the pressure distribution and web-to-roll gap profile spanning the coating bead. The effect of key operating parameters, web-to-roll speed ratio, web-tension, wrap-angle, capillary number and cell-geometry, on the degree of fluid pick-out from gravure cells and the coated film thickness is explored. Although an idealised model, the trends observed show qualitative agreement with existing experimental data collected on a small scale gravure coating rig and point the way forward to the eventual formulation of a full three-dimensional predictive model of the process.

Keywords: Films, Fluid Mechanics, Hydrodynamics, Mathematical Modelling, Coating Flows and Gravure Roll Coating. Mathematical Modelling And Simulation.
\end{abstract}

\section{Introduction}

Gravure roll coating in its various forms, while having its origins in the printing industry, is recognised within the coating community as a very useful and desirable technique for the production and deposition at high speeds of very thin films with a consistent level of uniformity [1]. Such coaters can be operated in forward or reverse mode as in the case of their rigid roll counterparts but there the similarity ends: the distinguishing features, and those that have made it less amenable to modelling, are that the surface of a gravure roll is engraved/etched with a discrete pattern of cells or grooves and the transfer of liquid from roll to substrate/web involves an element of elastrohydrodynamics. The latter is true whether liquid is transferred initially in the nipped region of an elastomer covered roll prior to meeting the substrate (off-set variant) or is simply deposited directly onto it (direct variant). Gravure rolls are manufactured in a variety of ways, either by knurling a roll surface against a hard steel master or by using a laser to engrave a ceramic layer surrounding a metal core. Accordingly, it is possible to achieve a wide range of patterns - discrete cells (for example, quadrangular indents) or continuous grooves (as seen on a tri-helical machined roll), as well as an increasingly popular class of surfaces containing discrete cells linked by small channels - such as Pamarco's QCH rolls.

The aim of the present work is to develop a multiscale mathematical model of discrete cell gravure coating, operating in reverse mode. This particular configuration

${ }^{1}$ email: r.w.hewson@leeds.ac.uk 


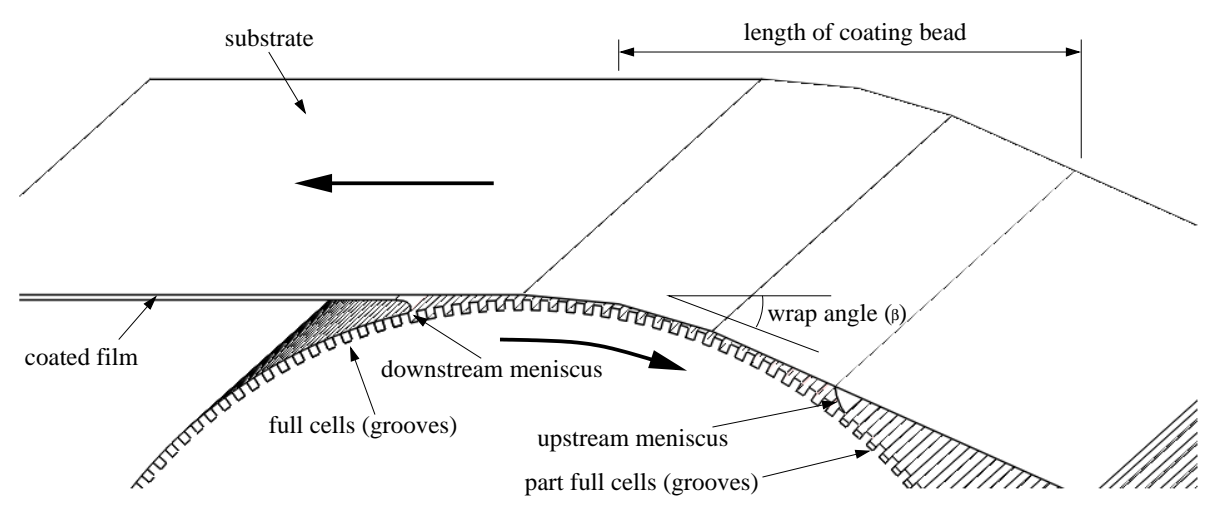

Figure 1: Schematic of a reverse gravure roll coating process showing the two dimensional cell (or axially aligned groove) arrangement investigated. The arrows indicate the direction of motion.

is chosen since direct-gravure coating is often the industrial method of choice, with the reverse mode of operation exhibiting a wider stable operating window [2] which is most likely attributable to the nature of the pressure gradient on the downstreamside of the coating bead. Also, it is important to note that some progress on the modelling front has been made in the related case of tri-helical gravure roll coating $[3,4,5]$, which involved solving equations for the flow along tri-helically cut grooves; the application of simplified meniscus descriptions upstream and downstream have shown that it is possible to predict reasonably well the coated film thickness deposited onto the web. For illustrative purposes a schematic of the process of interest is provided in figure 1.

At first sight the process, characterised by a coating bead which is long and thin, bounded from above and below by the web and roll surface, respectively and to the left and right by downstream and upstream menisci, appears to be an ideal candidate for analysis via lubrication theory. However, the nature of the roll surface represents a severe restricting factor with the cell or groove dimensions involved being an order of magnitude, or more, greater than the variable web-to-roll separation (gap). Until quite recently, analyses of the process have concentrated in the main on modelling the upstream meniscus region. The first such work was that of Rees [6] for the case of a single cell moving beneath a pinned meniscus. Subsequent models include those of Powell et. al. [7] and Schwartz et al. [8, 9]. The former derived a mathematical model for the partial evacuation of liquid from a rectangular cavity by the action of a passing meniscus; however, their two-dimensional time-dependent solutions of the governing Navier-Stokes equations required the imposition of the web-to-roll gap, web velocity and cross-cell pressure gradient in order to explore their influence on cell emptying. The latter authors considered the case of multiple cells, appealing to lubrication theory to examine the three-dimensional problem of liquid withdrawal from gravure cells; the effect of cell pattern is explored but is restricted to the flow domain beneath the upstream meniscus. Although both approaches fail to adequately replicate the real problem they reveal between them that: (i) an increase in cell size (width) and a decrease in Capillary number (ratio of viscous to surface tension forces) results in greater fluid pickout, $\phi$ (the ratio of volume of fluid leaving the coating bead to that entering via the cells); (ii) cell orientation has an effect on pickout. 
The recent related work of Yin \& Kumar $[10,11]$ considers the case of flow within a cavity bounded from above by a flexible surface of negligible thickness which can be argued at first sight is not too dissimilar to what occurs at the cell level of a gravure roll coater. These models are based on lubrication theory and Stokes flow conditions, and solved numerically. Their results suggest that the cell shape has a significant effect on the pressure profile which exists and the deformation of the flexible surface; also revealed via streamline plots are the various flow structures that transpire within the cavity. However, at a cellular level it has been shown to be an over simplification to ignore the thickness of the web, the stiffness of which results in negligible deformation on a length-scale commensurate with a single cell [12]; on the contrary, the web deformation that occurs on a length-scale typical of the coating bead itself is significant. Indeed, it is this observation that forms the basis of the multi-scale model presented below. Since the primary focus is that of modelling, references is made subsequently to relevant complementary experimental work only.

The mathematical model describing the problem to be investigated is formulated in section 2 , together with the method of solution. This is followed by a comprehensive set of results in section 3, with the findings summerised in section 4 .

\section{Mathematical Model and Method of Solution}

Consider the case of a discrete-cell direct gravure roll coater operating in reverse mode, with the simplifying features that the cells run the entire length of the roll, having a radius $R_{\text {roll }}$, which is assumed to be long enough so that the flow, with inertia neglected, in a cross-sectional plane sufficiently far enough away from its ends can be assumed two-dimensional. This is exactly the situation that would arise in the case of tri-helical gravure roll coating if the groove angle (measured normal to the roll axis) was set at $90^{\circ}$ [12]. The assumption of essentially two dimensional flow is supported by the difference in scale between the length of the gravure roll (of the order of meters) and the web-to-roll gap size that is dictated by bead pressure (of the order of $1-100 \mu \mathrm{m}$ ), which ensures that there can be no significant axial pressure gradients present. The same would be the case for discrete three dimensional cells where the roll geometry is periodic in the direction of the roll axis; however, for the case when there is a cell mesh angle viscous generated cross flow could exist.

The liquid forming the coating bead, which is bounded from above by the web, having speed $U_{w e b}$, and below by the roll surface, peripheral speed $U_{\text {roll }}$, is taken to be incompressible with constant viscosity $\mu$, density $\rho$ and surface tension $\sigma$. In addition, the length of the coating bead (of the order of centimeters), see Figure1, is assumed to be much greater than the thickness of the web (typically $10-200 \mu m$ ) it supports such that the latter can be considered as infinitely thin, the consequence being that the pressure distribution spanning the coating bead is balanced purely and simply by the tension in the curved web. This then forms the basis of the model formulated below - one which assumes the web to to be rigid and have a flat profile (nominally parallel to the roll surface) on the scale of the dimensions of a cell (small), while over the large scale of the entire bead the web is flexible and does not necessarily conform to the roll surface.

The problem, having two such disparate scales (at the cell and coating bead extremes), lends itself well to analysis via a top-down homogenisation approach such as the Heterogeneous Multiscale Method (HMM) described by E et al.[13] and E \& Engquist [14]. If in addition, as is the case here, the linear Stokes approximation is used to solve for the flow at the cell level, the number of variables involved is reduced 


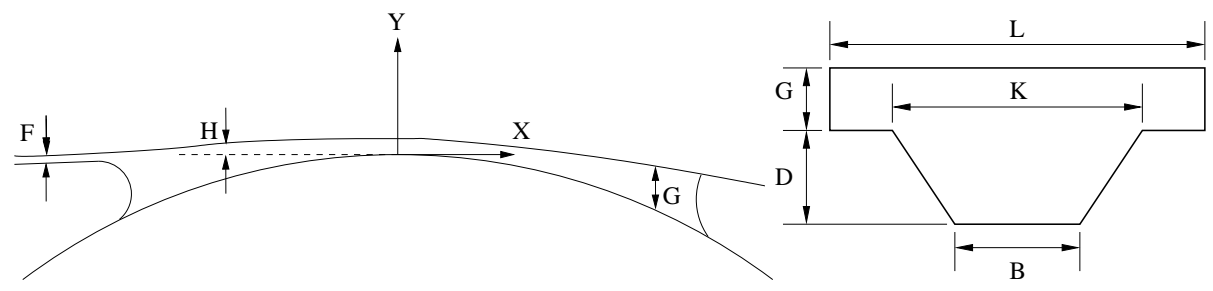

(a)

(b)

Figure 2: (a) Large scale and (b) small scale solution domain definitions.

significantly, making the serial coupling method described by E and colleagues an ideal means of extracting data at the small-scale for use at the large-scale.

The approach taken consists of two parts, corresponding to the two length-scales involved. The first obtains the homogenised pressure gradient-flow rate relationship (via the solution of the flow within a single cell); the second considers the entire coating bead. The solution domain, illustrated in figure 2(a), shows the coordinate system employed; $G(x)$ and $H(x)$ are the web-to-roll gap, and web deflection from the horizontal, respectively. The web-to-roll speed ratio as given by:

$$
S=\frac{U_{\mathrm{web}}}{U_{\mathrm{roll}}},
$$

is negative since the web and roll surface move in opposite directions as they traverse the coating bead.

All lengths, $\left(G, H, X, Y, R_{\text {roll }}\right)$, the pressure, $P$, web tension, $T$, coated film thickness $F$, and volume flow rate in the coating bead, $Q$, are non-dimensionalised as follows:

$\frac{\left(G, H, X, Y, R_{\mathrm{roll}, F}\right)}{K}=\left(g, h, x, y, r_{\mathrm{roll}}, f\right), p=\frac{P K}{\mu U_{\mathrm{roll}}}, \quad t=\frac{T}{\mu U_{\mathrm{roll}}} \quad$ and $\quad q=\frac{Q}{K U_{\mathrm{roll}}}$.

\subsection{Cell Length Scale Problem}

In order to formulate the large-scale problem, Figure 2(a), attention is first focused on deriving an appropriate relationship between the pressure gradient, flow rate and web-to-roll gap at a cellular level. This is done by considering a single cell, the solution domain and geometry for which is shown in Figure 2(b).

Choosing the length-scale $K$ as the cell opening defines a clear link between the two different scales governing the problem - the solution domain at the large-scale being two or more orders of magnitude larger. The non-dimensional cell depth and base-width are $d=\frac{D}{K}$ and $b=\frac{B}{K}$, respectively; the non-dimensional line-width is given by $l=\frac{L}{K}$.

The small scale of the flow problem allows the inertial terms to be neglected (as is typical in smooth roll coating analyses where lubrication theory is applied $[15,16$, 17]). The Stokes flow problem is solved over the domain of interest, Figure 2(b), subject to the following periodic boundary conditions $u_{\mathrm{lhs}}=u_{\mathrm{rhs}}, v_{\mathrm{lhs}}=v_{\mathrm{rhs}}$ and $p_{\mathrm{lhs}}=p_{\mathrm{rhs}}+\Delta p$, where $u, v$ are the velocities in the $x, y$ directions, $p$ is the pressure, and the subscripts throughout refer to the left hand (lhs) and right hand (rhs) boundaries. The $\Delta p$ term refers to the pressure difference across the domain, which when divided by its length $L$ gives a value for the averaged pressure gradient 


\begin{tabular}{|c|c|c|}
\hline Condition & $\frac{p_{\text {rhs }}-p_{\text {lhs }}}{l}\left[\approx \frac{d p}{d x}\right]$ & $S\left[=\frac{U_{\text {web }}}{U_{\text {roll }}}\right]$ \\
\hline 1 & -1 & -1 \\
2 & 0 & -1 \\
3 & 0 & 1 \\
\hline
\end{tabular}

Table 1: Gravure Cell Pressure Boundary Conditions.

$\frac{d p}{d x}$. The no-slip boundary condition is applied at the cell walls, the top wall (the moving web) is given a non-dimensional velocity of $S-1$; the -1 component is due to the change in the frame of reference from that of a moving roll and web in the laboratory frame of reference to that of a stationary roll surface. The flow rate, $q$, passing through the domain is found by converting back from the stationary roll frame of reference to that of the laboratory frame of reference. The values for $S$ and $\frac{d p}{d x}$ for which simulations are undertaken is given in table 1 . To obtain the flow rate, only conditions 1 and 2 need to be solved numerically, using Comsol Multiphysics, as the third solution is tractable analytically - based on the cross-sectional area of the cell and the web-to-roll gap - and results in zero fluid flow in the roll surface frame of reference. From the flow rates for the three conditions the following general pressure gradient-flow rate relationship can be written:

$$
q=A(g) \frac{d p}{d x}+B(g) S+C(g),
$$

where the coefficients $A(g), B(g)$ and $C(g)$ are functions of the web-to-roll gap, $g$, only. The linear nature of Stokes flow, ensures that the flow rate scales linearly with the web speed, $S$, and pressure gradient, $\frac{d p}{d x}$. Equation (3) can be rearranged to give:

$$
\frac{d p}{d x}=[\mathcal{F}(q, g, S)]=\frac{q-B(g) S-C(g)}{A(g)} .
$$

The term $[\mathcal{F}(q, g, S)]$ used in the large scale simulation is a linear function of the speed ratio and pressure gradient. The small scale solution therefore only needs to be found over a range of web-to-roll gaps in order to obtain the terms $A(g)$, $B(g)$ and $C(g)$. If the problem was that of a smooth roll coating process, $d \rightarrow 0$, (indicated here by the subscript $L$ ) the function $F(q, g)_{L}$ and the coefficients $A(g)_{L}$, $B(g)_{L}$ and $C(g)_{L}$ would be identical to those arising from lubrication theory, namely $A(g)_{L}=-\frac{g^{3}}{12}, B(g)_{L}=\frac{g}{2}$ and $C(g)_{L}=-\frac{g}{2}$. Plotting, as in figure 3 , the ratio of the coefficients for a typical gravure cell geometry $(l=1.2, b=0.7)$ to those associated with the the lubrication approximation, it is clear that as the cell depth increases, the divergence between the computed values similarly increases.

\subsection{Large Scale Problem Formulation}

From the assumption, derived from the linearity of Stokes flow, that the pressure gradient within the solution domain is a function of flow rate and web-to-roll gap only:

$$
\frac{d p}{d x}=\mathcal{F}(q, g, S),
$$

while conservation of mass requires that:

$$
\frac{d q}{d x}=0 .
$$




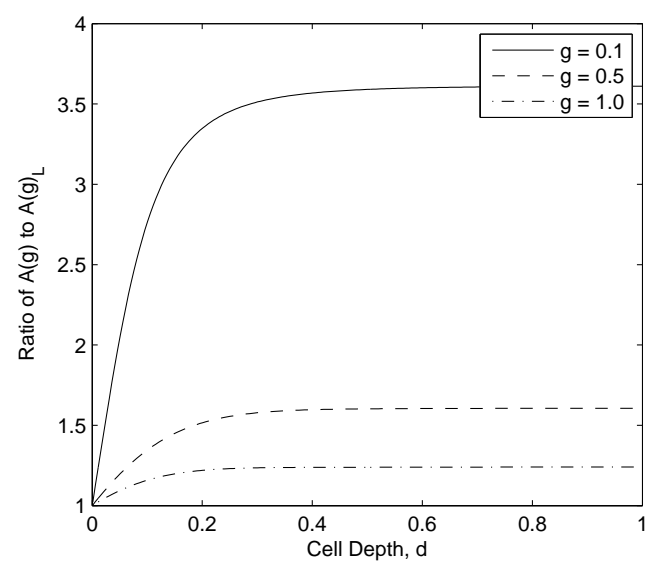

(a)

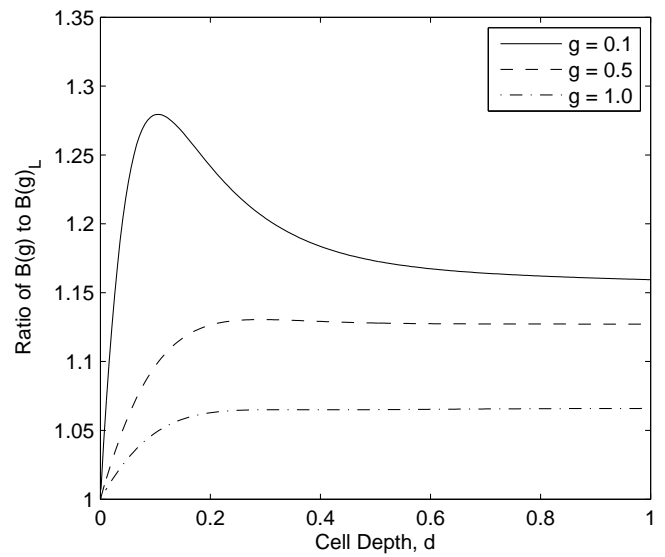

(b)

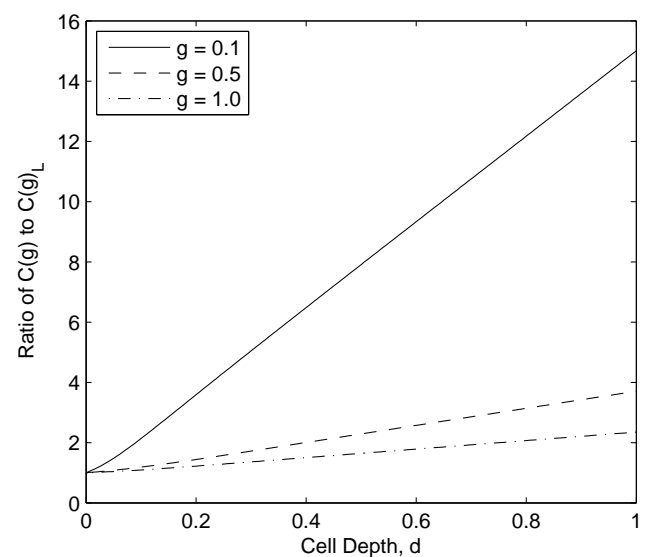

(c)

Figure 3: Plot of the ratio of the coefficients, $A(g), B(g)$ and $C(g)$, for a typical gravure roll cell geometry $(l=1.2, b=0.7)$ to their lubrication theory counterparts, $\frac{g^{3}}{12}, \frac{g}{2}$ and $\frac{-g}{2}$ for different $g$, showing the divergence between them as the cell depth, $d$, increases. 
Since it is assumed that the web deflection, $h$, on the length scale of the coating bead, depends only on the web tension, $t$, the pressure at a point within the coating bead can be related to the web's local radius of curvature there, namely:

$$
p=\frac{t \frac{d^{2} h}{d x^{2}}}{\left(1+\left(\frac{d h}{d x}\right)^{2}\right)^{\frac{3}{2}}} .
$$

A considerable complication with regard to solving the above equations is that the location of the bounding upstream and downstream menisci (and therefore the extents of the solution domain) is not known a priori. Accordingly, an ad hoc moving mesh approach is adopted to specify the domain, the $x$ dimension being governed by the auxiliary variable, $\zeta$, such that:

$$
\frac{d^{2} x}{d \zeta^{2}}=0
$$

which upon integration leads to the following relationship:

$$
\frac{d x}{d \zeta}=\Psi
$$

with $\Psi$ dependent on the location and type of the upstream and downstream boundary conditions.

Therefore, the set of six first order ordinary differential equations required to solve the problem in terms of $\zeta$ are:

$$
\begin{aligned}
& \frac{d p}{d \zeta}\left[=\Psi \frac{d p}{d x}\right]=\Psi F(q, g), \quad[\text { from equation }(5)] \\
& \frac{d q}{d \zeta}\left[=\Psi \frac{d q}{d x}\right]=0, \quad[\text { from equation }(6)] \\
& \frac{d}{d \zeta}\left(\frac{d h}{d x}\right)\left[=\Psi \frac{d}{d x}\left(\frac{d h}{d x}\right)\right]=\Psi \frac{p}{t}\left(1+\left(\frac{1}{\Psi} \frac{d h}{d \zeta}\right)^{2}\right)^{\frac{3}{2}}, \quad \text { [from equation (7)] } \\
& \frac{d h}{d \zeta}\left[=\Psi \frac{d h}{d x}\right]=\Psi \frac{d h}{d x}, \quad[\text { from equation }(7)] \\
& \frac{d}{d \zeta}\left(\frac{d x}{d \zeta}\right)\left[=\Psi \frac{d}{d x}\left(\frac{d x}{d \zeta}\right)\right]=0, \quad[\text { from equation (8)] } \\
& \frac{d x}{d \zeta}\left[=\Psi \frac{d x}{d x}\right]=\Psi . \quad[\text { from equation (9)]. }
\end{aligned}
$$

The web-to-roll gap, $g$, is obtained in terms of the web location, $h$, and a parabolic approximation in $x$ of the roll surface:

$$
g=h+\frac{x^{2}}{2 r_{\text {roll }}} .
$$

In order to close the problem six boundary conditions are required, one each at the upstream (us) and downstream ( $d s$ ) for: (a) the meniscus pressures; (b) the web wrap angle at the meniscus locations; (c) the menisci locations themselves. There is also the matter of specifying values for the three dynamic contact angles which characterise the coating bead, which for simplicities sake are all taken to be $90^{\circ}$. Accordingly, the meniscus pressures are given by:

$$
\begin{aligned}
& p_{\mathrm{us}}=\frac{1}{C a} \frac{1}{g}, \\
& p_{\mathrm{ds}}=\frac{1}{C a} \frac{2}{g} .
\end{aligned}
$$


where $C a=\frac{\mu U_{\text {roll }}}{\sigma}$ is the capillary number.

Relating the gradient in $h$ to the wrap angle, $\beta$, gives:

$$
\begin{aligned}
& \left.\frac{d h}{d x}\right|_{\text {us }}=-\beta, \\
& \left.\frac{d h}{d x}\right|_{\mathrm{ds}}=0 .
\end{aligned}
$$

The meniscus locations are specified using the Bretherton equation [18] (based on the same asymptotic analysis as Landau \& Levich [19] - see also [20]):

$$
\begin{aligned}
& x_{\mathrm{us}}=-\sqrt{2 r_{\mathrm{roll}}\left(\frac{q}{1.34 C a^{\frac{3}{2}}}-h\right)}, \\
& x_{\mathrm{ds}}=\sqrt{2 r_{\mathrm{roll}}\left(\frac{2 q_{2}}{1.34(S C a)^{\frac{3}{2}} S}-h\right)} .
\end{aligned}
$$

where $q_{2}=q_{\text {in }}-q$ and $q_{\text {in }}$ is simply the volume flow rate into the coating bead - based on the cell geometry; this results in $q_{2}$ being the volume flow rate of fluid leaving the coating bead on the web. Note that in the case of high capillary numbers it is possible to modify the above expressions for pressure and meniscus location accordingly - see, for example, [21] or [22].

Equations (10) to (13) were solved subject to the above boundary conditions via Matlab using the boundary value problem (BVP) solver BVP $4 c$ (the ease with which the full equation for web tension, equation ( 7$)$, can be implemented in the non-linear Matlab solver is the reason for its inclusion in the governing equations rather than assuming a small web gradient and setting the denominator of equation (7) to one). The equation set is extremely stiff and its solution somewhat problematic in that only a small region of the parameter space can be solved ab initio. However, once such a solution has been obtained for one set of parameters continuation can be used to incrementally change one parameter to obtain the next solution.

\section{Results}

Before examining the effects of the key operating parameters $(S, C a, \beta$, web tension and cell geometry) attention is focused on the results obtained for a typical set of values, as shown in figure 4. Figure 4a shows the profile of the web and roll surfaces; figure $4 \mathrm{~b}$ shows the change in the web-to-roll gap, revealing a region of near constant gap from $-100<x<100$. This corresponds to the near constant positive pressure across the same region as shown in figure 4c. The constant pressure within this region of the coating bead alludes to the insensitivity of pickout and coated film thickness to wrap angle - as discussed subsequently.

It is interesting to note also the variation in pressure and web shape near the downstream meniscus is similar to that found in the nip-region of a deformable twin roll coater, in which either or both is covered with a thin elastomer layer [23]. The order of the minimum web-to-roll gap is around one tenth of the cell opening length, $K$, corresponding to gaps of around $20 \mu \mathrm{m}$ for cell sizes typical of those used industrially. In actuality the roughness of gravure roll lands can be of the same order as the web-to-roll gap; if such asperities exist it points to a potential mechanism for scratching of the web [24]. The prediction of the web-to-roll gap therefore alludes 


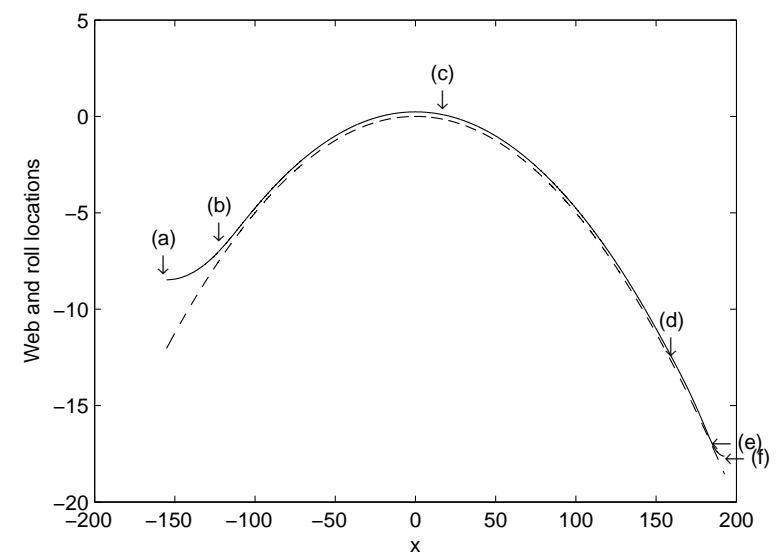

(a)

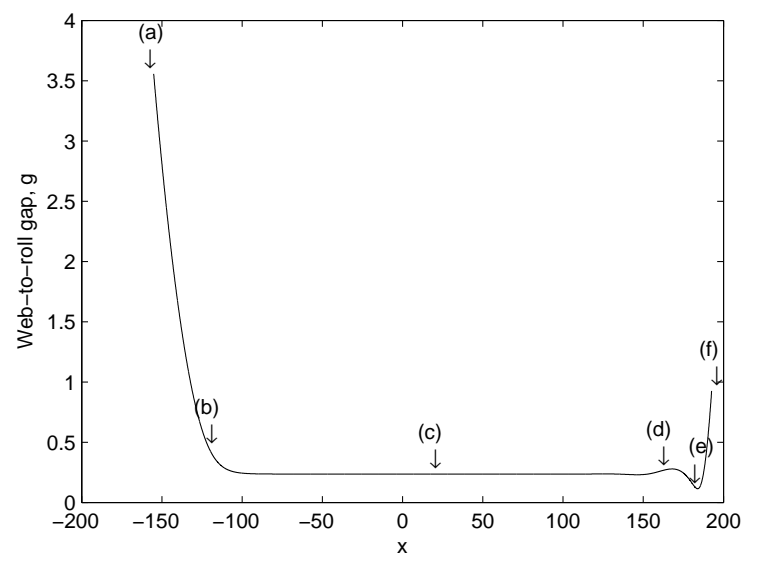

(b)

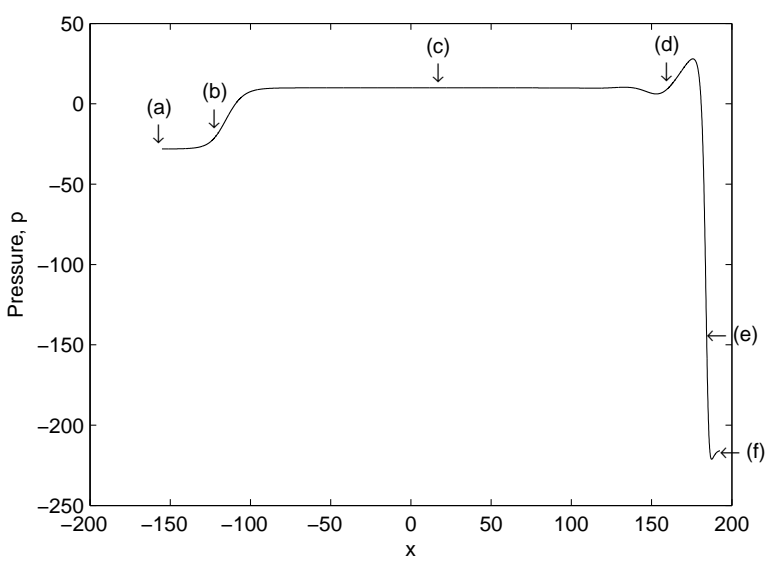

(c)

Figure 4: Typical results for (a) the web and roll geometry (roll surface is the broken line), (b) the web-to-roll gap and (c) the pressure within the coating bead for the following parameters; $S=1, R=1000, C a=0.01, \beta=0^{\circ}, t=10,000, l=1.5$, $d=0.5$ and $b=0.5$. The labels on the graphs refer to figures $5(\mathrm{a})$-(f). 


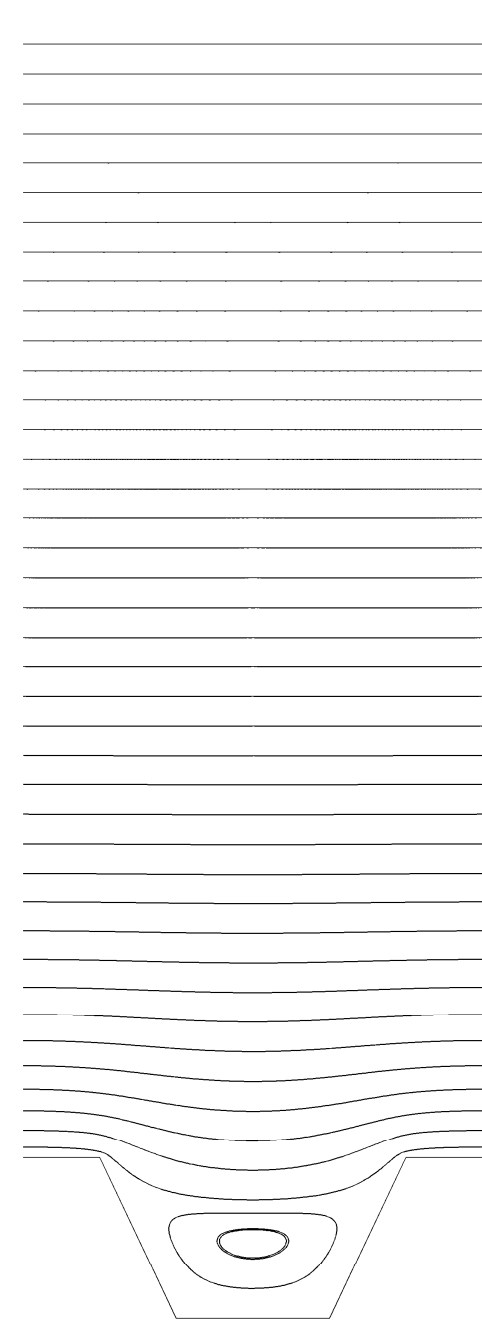

(a)

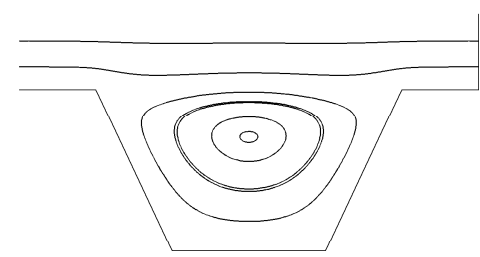

(c)

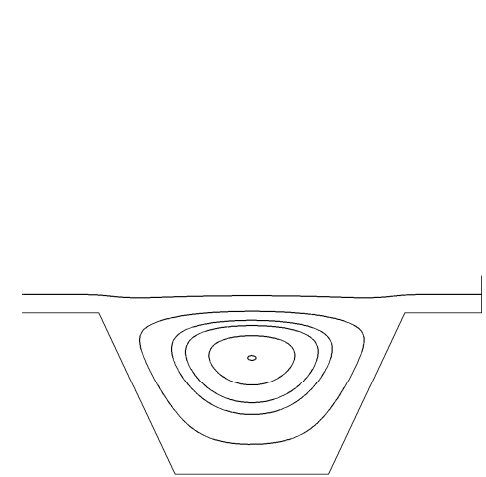

(e)

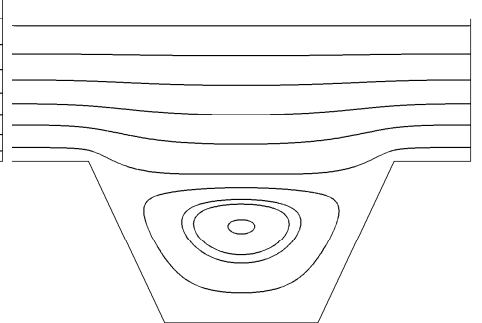

(b)

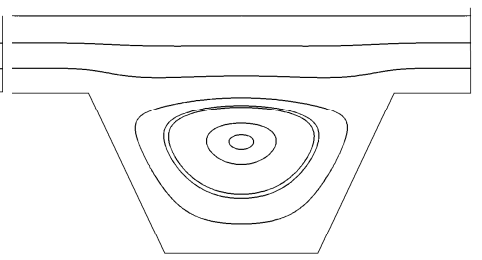

(d)

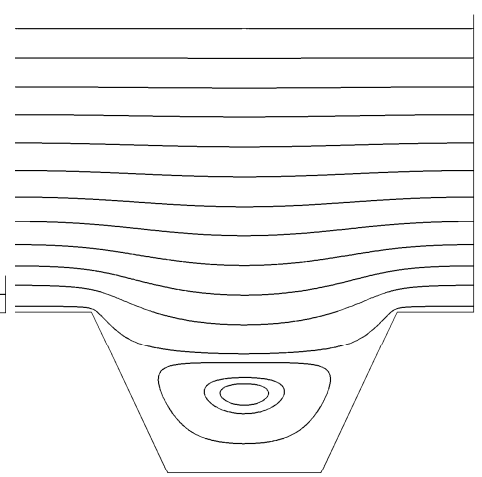

(f)

Figure 5: Cell streamlines at different locations throughout the coating bead, as indicated in figure 4 
to the maximum roll surface roughness beyond which scratching of the web would be expected.

The streamline patterns given in figure 5 reveal the flow features present at the small scale. It is interesting to note that the size of the eddy which exists in each case varies with the web-to-roll gap (and the resulting pressure gradient across the cell). It provides a clear indication that through the careful design of the cell geometry it may be possible to eliminate the eddy at some location in the coating bead, thus reducing the possibility of solidification of the coating fluid and avoiding the accumulation of debris in the roll cells. The study and control of such eddies has been the focus of attention of a number of investigators (see for example [25]). The elimination of eddies for the problem of interest is clearly an area worthy of further investigation and could be used to optimise gravure roll design.

\subsection{Operating Parameters}

The two dimensional nature of the simple model means that direct quantitative comparison with complementary existing experimental data is not possible; however, the data of Kapur [26, 24] facilitates qualitative comparisons.

Of particular interest are the results showing the change in the pickout and coated film thickness with speed ratio. One such result is shown in figure 6; as expected, due to the increased viscous forces exerted by the web, as speed ratio increases so too does pickout. The relationship between speed ratio and coated film thickness is more complex, this is because there are two opposing effects as the speed ratio increases: (i) that of a rise in pickout which increases the volume flow rate of coating fluid leaving on the web; (ii) the increase in the web surface area passing through the coating bead per unit time, leading to an increase in area to be coated per second, which in turn leads to a decrease in the coated film thickness. For the operating conditions and cell geometry considered the coated film thickness increases with speed ratio, up to a maximum at $S \approx 1.7$, before decreasing. Qualitative agreement with the results of Kapur [26, 24] is good (figure 7); he noted that for low speed ratios pickout increases quadratically with speed ratio before a more linear relationship prevails, this leads to an increase in coated film thickness for low speed ratios before a constant coated film thickness is reached. In agreement with this, the model predicts the initial rise in coated film thickness (and corresponding increase in pickout); beyond which lies a gradual decrease in coated film thickness (outside the experimental range examined by Kapur). Indeed if one considers a roll operating at constant speed there is a constant fluid volume entering the coating bead; therefore, as the pickout has an upper bound of one the coated film thickness must decrease as this limit is approached. The reason for the low pickout at low speed ratios is most likely due to the higher volume of fluid entering the coating bead, because of the two dimensional nature of the problem, than would otherwise be observed in the case of a discrete three dimensional cell patternation.

The effect of capillary number is also considered; figure 8 shows that as capillary number increases there is an initial rapid increase in pickout which levels out as pickout becomes more insensitive to changes in capillary number. This increase in pickout with capillary number is clearly observable in the results of Kapur, who found that pickout and film thickness increased with capillary number. Wrap angle insensitivity, alluded to by figure 4c, was confirmed by examining a range of wrap angles from $\beta \in\left[0^{\circ}, 5^{\circ}\right]$. Examining the pressures within the coating bead highlights the near zero pressure gradient across the region over which the web is wrapped around the roll. Again this insensitivity has been observed experimentally by Kapur 


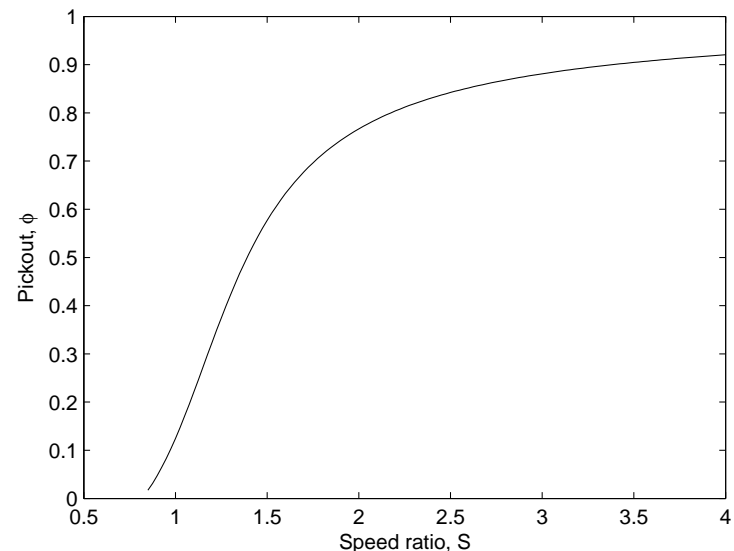

(a)

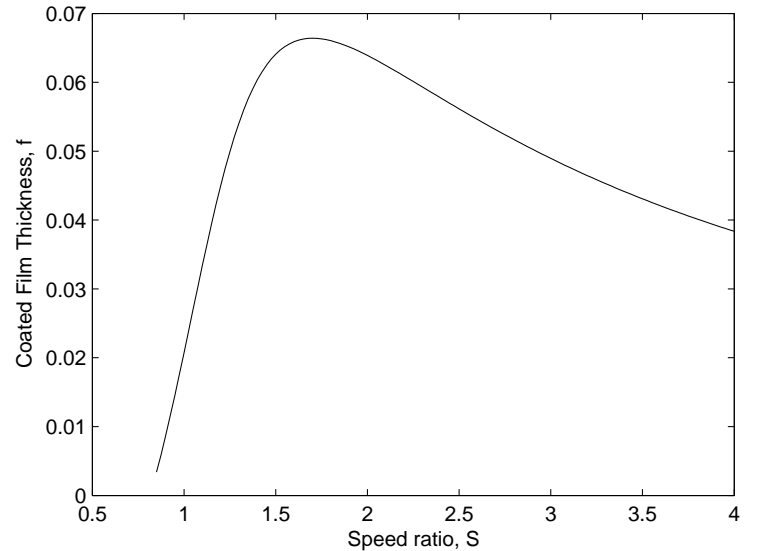

(b)

Figure 6: Pickout and coated film thickness as a function of speed ratio.

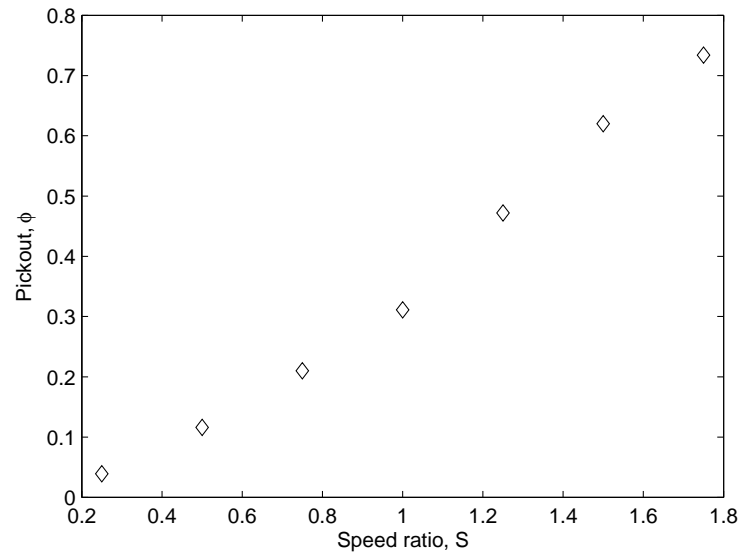

(a)

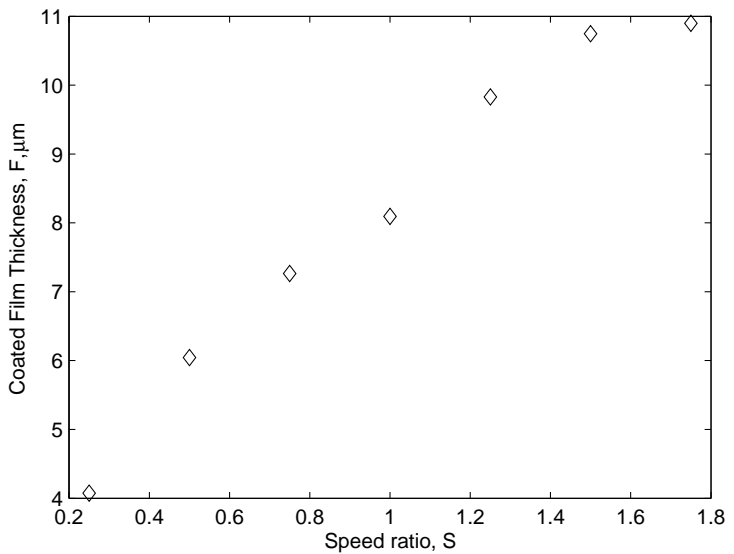

(b)

Figure 7: Typical experimental pickout and film thickness results obtained by Kapur [24] $(\mu=0.00409$ Pas and $\sigma=0.033 \mathrm{~N} / \mathrm{m})$.

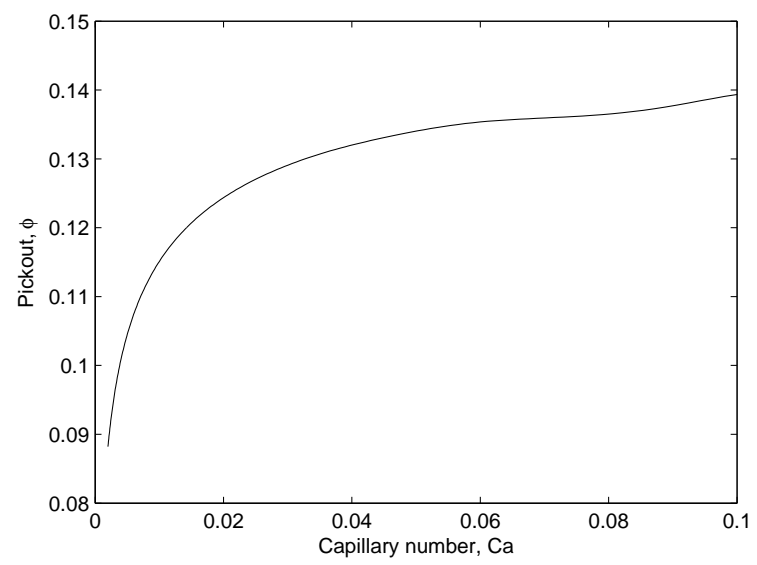

Figure 8: Pickout as a function of capillary number. 


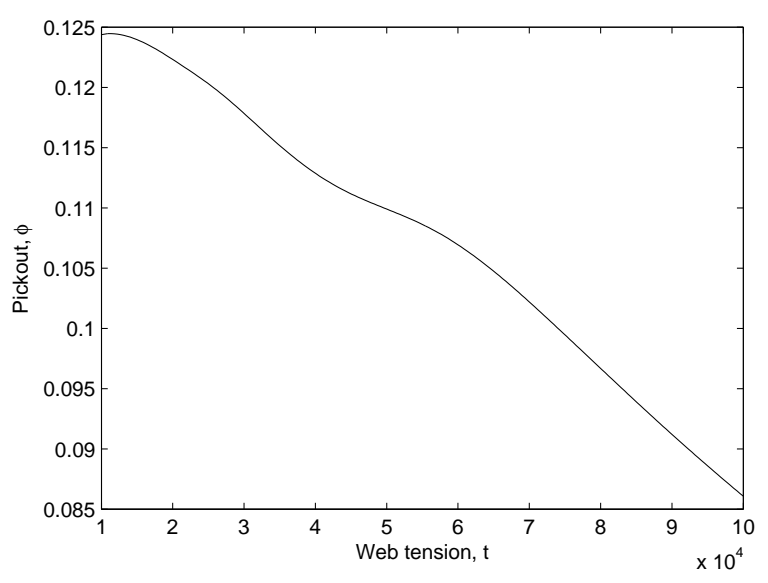

Figure 9: Pickout as a function of web tension

as well as by Benkreira \& Patel [27]. The sensitivity to web tension is shown in figure 9 . The range of web tensions examined experimentally by Kapur cover $500-$ $2500 \mathrm{Nm}^{-1}$; over such a five fold increase in tension the pickout predicted by the model changes by only around $10 \%$ and may not have been noticable in these earlier experiments.

\section{Roll Dimensions}

Examining the effect of changing cell depth is shown in figure 10. It can clearly be seen that increasing the cell depth, $d$, leads to a decrease in the pickout, $\Psi$; this is as expected and as was observed by Kapur who found that shallow cells exhibited a greater pickout than deeper ones. Turning to the coated film thickness the opposite trend can be observed, an increase in cell depth leads to an increase in the coated film thickness deposited onto the moving web; although increasing cell depth leads to a decrease in pickout the volume of fluid entering the coating bead is greater, leading to an increase in coated film thickness. For the same reason, increasing the cell base length, $b$ (as shown in figure 11) leads to a decrease in pickout and an increase in film thickness. Figure 12 shows that by changing the line width, $l$, while keeping all other parameters constant predicts that pickout falls as line width increases; this in turn leads to an even more pronounced reduction in film thickness due to the reduced amount of fluid entering the coating bead. 


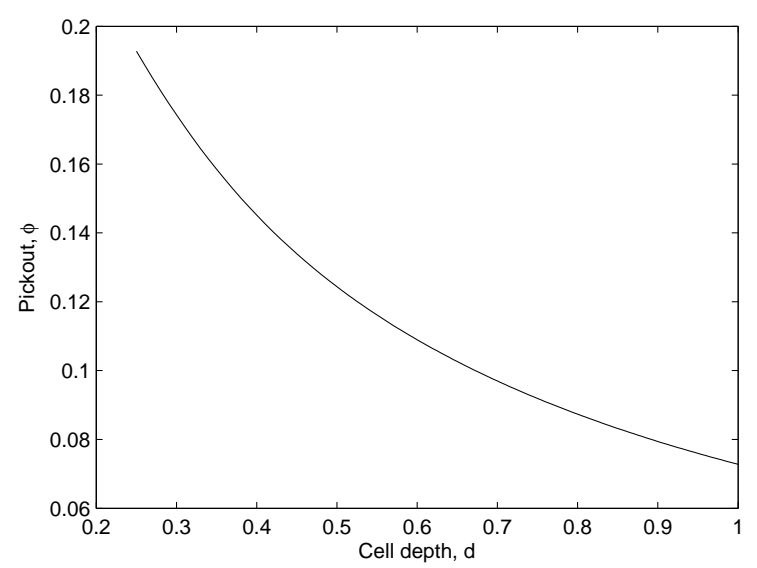

(a)

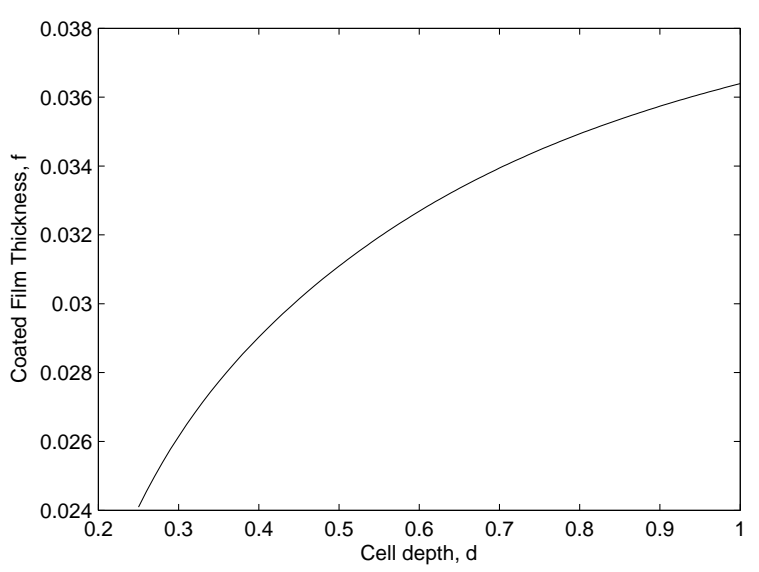

(b)

Figure 10: Pickout and coated film thickness as a function of cell depth.

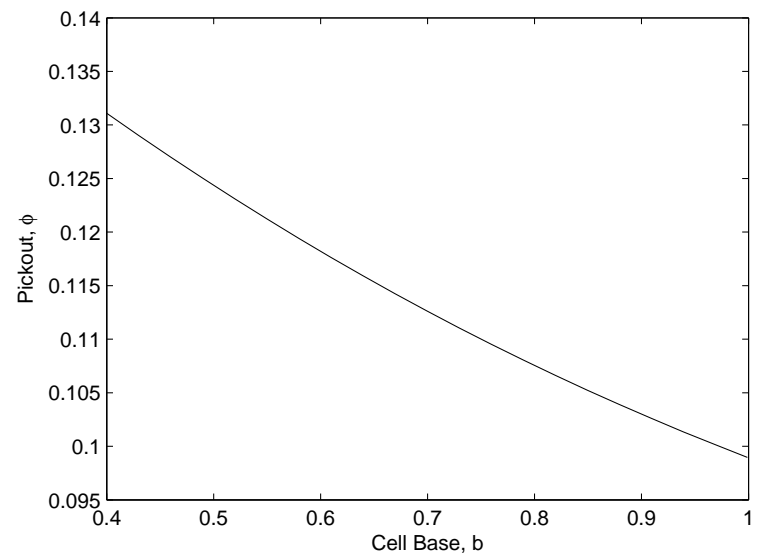

(a)

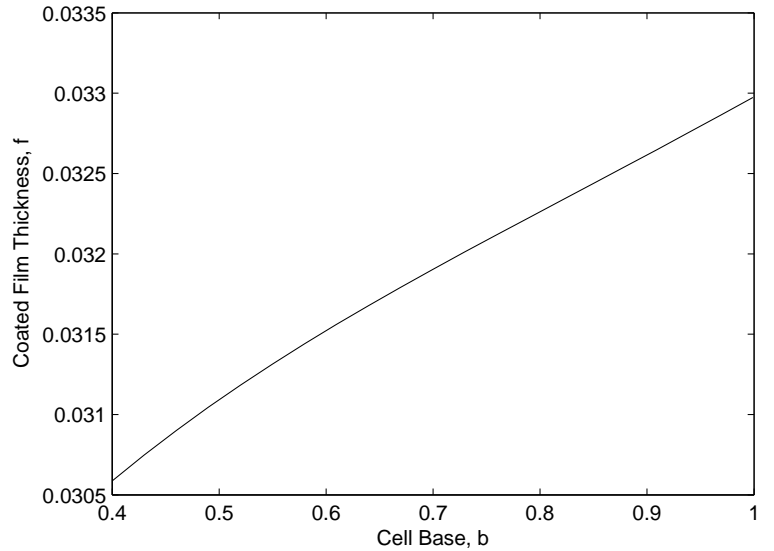

(b)

Figure 11: Pickout and coated film thickness as a function of cell base length.

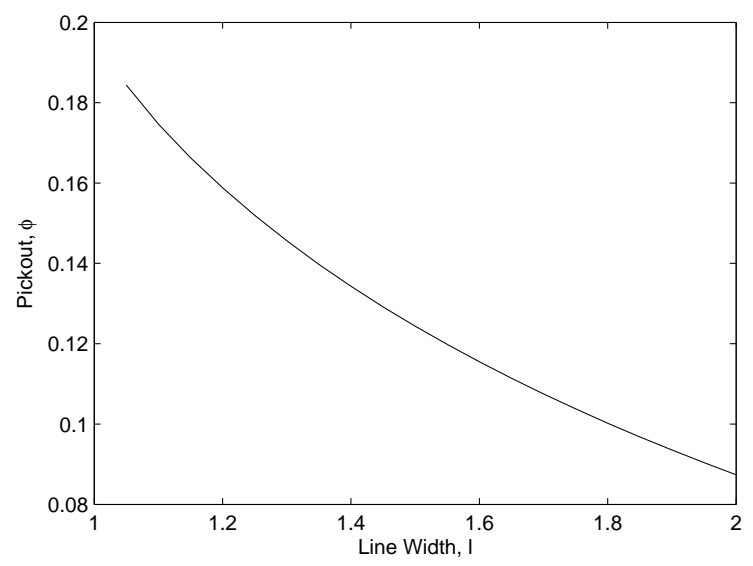

(a)

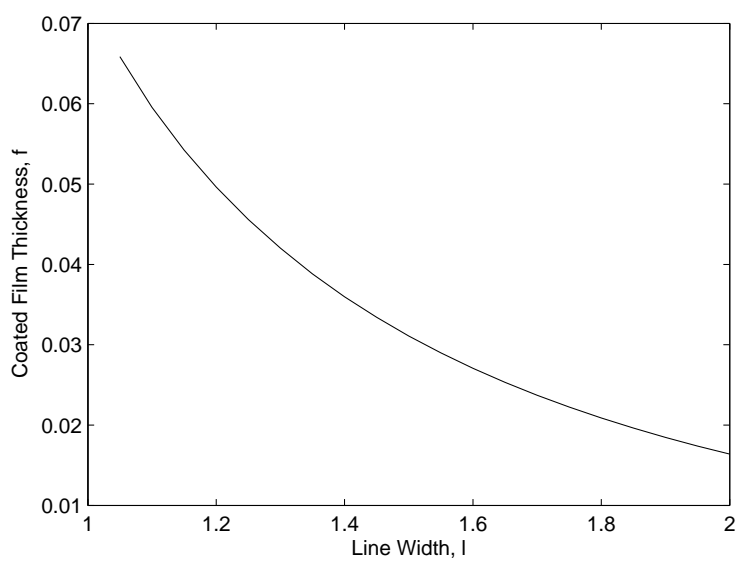

(b)

Figure 12: Pickout and coated film thickness as a function of line width. 


\section{Conclusion}

A two dimensional discrete cell gravure roll coating model has been derived, the predictions from which show qualitative agreement with the observations and experimental data of Kapur [24] obtained on a laboratory scale gravure roll coater. The novel approach adopted is able to predict the global coating performance (such as pickout and coated film thickness) as well as the flow features on the small scale. The application of such a multiscale approach to three-dimensional cells will be the topic of future work. An in depth study of the flow features occurring at a cellular length scale is also of considerable interest. The interrogation of this small scale flow has important implications in relation to the presence or elimination of eddies within cells, the flow into and out of the eddy regions (bottom of the cells) and will ultimately have a direct impact on predicting coating defects and the solidification of coating fluid in the cells.

Investigation of the effect of operating conditions and cell geometry together with the relative importance of the parameters examined, enables the following conclusions to be drawn:

- The most important operating parameter is confirmed as being the web-toroll speed ratio: an increase in speed ratio leads to an increase in pickout; the resulting dependence of the coated film thickness is more complex.

- An increase in capillary number leads to an increase in pickout (and coated film thickness).

- An increase in web tension leads to a small decrease in pickout (and coated film thickness).

- The effects of cell geometry is clearly demonstrated: (i) shallower cells lead to a greater degree of pickout; (ii) deeper grooves lead to a larger coated film thickness; (iii) pickout decreases as the cell base increases;(iv) coated film thickness increases as the cell base increases; (v) both pickout and coated film thickness decrease as the line width (distance between cells) increases.

\section{References}

[1] S. F. Kistler and P. M. Schweizer. Liquid Film Coating - Scientific Principles And Their Technological Implications. Chapman and Hall, London, 1997.

[2] H. Benkreira and O. Cohu. Direct forward gravure coating on unsupported web. Chemical Engineering Science, 53(6):1223-1231, 1998.

[3] R. W. Hewson. Investigation Into Tri-Helical Direct Gravure Coating. PhD thesis, University of Leeds, 2006.

[4] R. W. Hewson, N. Kapur, and P. H. Gaskell. A theoretical and experimental investigation of tri-helical gravure roll coating. Chemical Engineering Science, 61(16):5487-5499, 2006.

[5] R.W. Hewson, N. Kapur, and P. H. Gaskell. Tri-helical gravure roll coating. Chemical Engineering Science, 65(4):1311-1321, 2010.

[6] S. R. Rees. An experimental and theoretical investigation of gravure roll coating. $\mathrm{PhD}$ thesis, University of Leeds, 1995. 
[7] C. A. Powell, M. D. Savage, and P. H. Gaskell. Modelling the meniscus evacuation problem in direct gravure coating. Chemical Engineering Research and Design, 78(A1):61-67, 2000.

[8] L. W. Schwartz, P. Moussalli, P. Campbell, and R. R. Eley. Numerical modelling of liquid withdrawal from gravure cavities in coating operations. Chemical Engineering Research and Design, 76(A1):22-28, 1998.

[9] L. W. Schwartz. Numerical modeling of liquid withdrawal from gravure cavities in coating operations; the effect of cell pattern. Journal of Engineering Mathematics, 42(3-4):243-253, 2002.

[10] X. Yin and S. Kumar. Lubrication flow between a cavity and a flexible wall. Physics of Fluids, 17(6), 2005.

[11] X. Yin and S. Kumar. Flow visualization of the liquid-emptying process in scaled-up gravure grooves and cells. Chemical Engineering Science, 61(4):11461156, 2006.

[12] R. W. Hewson, N. Kapur, and P. H. Gaskell. Modelling the discrete-cell gravure roll coating process: A new perspective. European Physical Journal Special Edition (European Coating Symposium 2007), 166(1):99-102, 2009.

[13] W. E, B. Engquist, and Z. Y. Huang. Heterogeneous multiscale method: A general methodology for multiscale modeling. Physical review B, 67(9):092101, 2003.

[14] W. E and B. Engquist. The heterogeneous multi-scale method for homogenization problems. In B. Engquist, P. Lotstedt, and O. Runborg, editors, Multiscale Methods in Science and Engineering. Springer, New York, 2005.

[15] P. H. Gaskell, M. D. Savage, J. L. Summers, and H. M. Thompson. Modelling and analysis of meniscus roll coating. Journal of Fluid Mechanics, 298:113-137, 1995.

[16] P. H. Gaskell, S. R. Rees, M. D. Savage, and S. P. Storey. A mathematical model of roll-to-web kiss coating. Chemical Engineering Research and Design, 76(A1):29-37, 1998.

[17] J. L. Summers, H. M. Thompson, and P. H. Gaskell. Flow structure and transfer jets in a contra-rotating rigid-roll coating system. Theoretical and Computational Fluid Dynamics, 17(3):189-212, 2004.

[18] F.P. Bretherton. The motion of long bubbles in tubes. Journal of Fluid Mechanics, 10:166, 1961.

[19] L. Landau and B. Levich. Dragging of a liquid by a moving plate. ACTA Physicochimica URSS, 17:42, 1942.

[20] L. W. Schwartz, H. M. Princen, and A. D. Kiss. On the motion of bubbles in capillary tubes. Journal of Fluid Mechanics, 172:259-275, 1986.

[21] K. J. Ruschak. Boundary-conditions at a liquid air interface in lubrication flows. Journal of Fluid Mechanics, 119(JUN):107-120, 1982.

[22] R. W. Hewson, N. Kapur, and P. H. Gaskell. A model for film-forming with newtonian and shear-thinning fluids. Journal of Non-Newtonian Fluid Mechanics, 162(1-3):21-28, 2009. 
[23] M. J. Gostling, M. D. Savage, A. E. Young, and P. H. Gaskell. A model for deformable roll coating with negative gaps and incompressible compliant layers. Journal of Fluid Mechanics, 489:155-184, 2003.

[24] N. Kapur. A parametric study of direct gravure coating. Chemical Engineering Science, 58(13):2875-2882, 2003.

[25] M. Scholle, A. Haas, N. Aksel, M. C. T. Wilson, H. M. Thompson, and P. H. Gaskell. Eddy genesis and manipulation in plane laminar shear flow. Physics of Fluids, 21(7), 2009.

[26] N. Kapur. Flow phenomena in fixed-gap and gravure roll coating systems. PhD thesis, University of Leeds, 1999.

[27] H. Benkreira and R. Patel. Direct gravure roll coating. Chemical Engineering Science, 48(12):2329-2335, 1993. 